\section{A regulatory circuit conferring varied flowering response to cold in annual and perennial plants}

\author{
Youbong Hyun, Coral Vincent, Vicky Tilmes, Sara Bergonzi* ${ }^{*}$ Christiane Kiefer $\dagger$, \\ René Richter, Rafael Martinez-Gallegos, Edouard Severing, George Coupland $\ddagger$
}

\begin{abstract}
The reproductive strategies of plants are highly variable. Short-lived annuals flower abundantly soon after germination, whereas longer-lived perennials postpone and spatially restrict flowering. We used CRISPR/Cas9 and interspecies gene transfer to understand divergence in reproductive patterns between annual and perennial crucifers. We show that in perennial Arabis alpina, flowering in response to winter cold depends on the floral integrator SQUAMOSA PROMOTER BINDING PROTEIN-LIKE 15 (SPL15), whose activity is limited to older shoots and branches during cold exposure. In annuals, this regulatory system is conserved, but cold-induced flowering occurs in young shoots, without requirement for SPL15, through the photoperiodic pathway when plants return to warm. By reconstructing the annual response in perennials, we conclude that characteristic patterns of reproduction in annuals and perennials are conferred through variation in dependency on distinct flowering pathways acting in parallel.
\end{abstract}

\footnotetext{
F
} loral induction from environmental cues synchronizes plant reproduction to changing seasons (1). However, the ability of an individual plant to respond to permissive environmental cues also depends on its developmental stage. This interaction between environmental and developmental processes varies among species, creating diversity in reproductive strategy. Monocarpic annuals are short-lived and flower in response to permissive seasonal cues soon after germination. By contrast, flowering of perennials is delayed, enabling sufficient resource accumulation to sustain their repeated life cycle (2). Moreover, perennials spatially restrict flowering, so that vegetative branches are retained after reproduction, implementing polycarpic life history. Perennial Brassicaceae species, including Arabis alpina, express two systems that limit reproduction in time and space. The first involves orthologs of the Arabidopsis thaliana MADS box transcription factor FLOWERING LOCUS C (FLC), called PERPETUAL FLOWERING 1 (PEP1) in A. alpina (3-7). In perennials, repression of FLC orthologs in winter cold enables flowering to occur through a process called vernalization, whereas their reactivation in warm restricts flowering. The second system, based on microRNA156 (miR156), acts in young meristems to prevent the flowering response to vernalization, but in older meristems, transcriptional repression of MIR156 allows the vernalization response to proceed $(6,8)$. We show

Max Planck Institute for Plant Breeding Research, Carl-vonLinne-Weg 10, D50829, Germany.

*Present address: Laboratory of Plant Breeding, Department of Plant Science, Wageningen-UR, Netherlands. †Present address: Center for Organismal Studies Heidelberg, Department of Biodiversity and Plant Systematics, Im Neuenheim Feld 345 69120, Heidelberg, Germany.

‡Corresponding author. Email: coupland@mpipz.mpg.de that the PEP1 and miR156 systems are mechanistically linked in shoot meristems by means of the flowering promoting factor SQUAMOSA PROMOTER BINDING PROTEIN-LIKE 15 (SPL15). Furthermore, we demonstrate that the extent to which plants depend on this pathway differs between annuals and perennials, conferring variation in the age at which plants respond to vernalization.

In diverse plant lineages, miR156 levels gradually decrease as plants age (9-11). In A. alpina, neither age nor miR156 affects PEP1 down-regulation during vernalization $(8,12)$, suggesting that agerelated vernalization is determined downstream of PEP1. Genome-wide studies in A. thaliana and $A$. alpina showed that FLC and PEP1 bind to corresponding regions in the promoters of SPL15 and AaSPL15, respectively (13-15). SPL15 mRNA is targeted by miR156, and in A. thaliana, SPL15 promotes flowering under short days $(16,17)$. The binding of PEP1 to AaSPL15 was confirmed by means of chromatin immunoprecipitationquantitative polymerase chain reaction (fig. S1), and pep1 mutants exhibited increased AaSPL15 PEP1 binding for AaSPL15 transcription (fig. S1) and supporting the effect of FLC detected in A. thaliana and Cardamine hirsuta $(13,18)$. Moreover, when older ( 6 weeks old) plants were exposed to vernalization, AaSPL15 mRNA increased at the shoot apical meristem (SAM), correlating with PEP1 repression and flowering (Fig. 1, A and B, and fig. S1) (3). However, vernalization was insufficient to promote AaSPL15 expression at the SAM of young plants (2 weeks old) that contain high miR156 levels (Fig. 1, C and D, and fig. S1) (8). Thus, AaSPL15 potentially integrates cues derived from winter cold and age of the plant at the SAM of A. alpina. mRNA levels, demonstrating the relevance of
To test whether AaSPL15 is involved in vernalization, mutations that inactivated AaSPL15 were induced in A. alpina accession Pajares (Paj) by using CRISPR/Cas9 (Fig. 1E and fig. S2) (19). Resequencing the genome of Aaspl15 mutants, we detected no mutations at potential off-target sites of the single-guide RNA (sgRNA) (fig. S3 and table S1). The isolated Aaspl15 mutants were less sensitive to vernalization compared with Paj. Most 5- and 6-week-old Aaspl15 mutants failed to flower after vernalization, whereas wildtype Paj were fully responsive at both ages (Fig. 1, F and G). Around 40\% of Aaspl15 individuals did produce flowers after vernalization; however, their inflorescences rapidly reverted to producing leaves (Fig. 1, F to I), suggesting that the mutant meristem was not committed to flowering. Similarly, loss of AaSPL15 activity delayed the expression of floral meristem identity gene AaLEAFY (AaLFY) during vernalization and the flowering of pep1 mutant in warm long days (fig. S4). The mutation cosegregated with the effect on vernalization response (fig. S5) and was complemented by a wtAaSPL15-VN transgene in which the wild-type AaSPL15 locus was fused to the fluorescent protein VENUS (fig. S6). These analyses demonstrate that AaSPL15 is required for the flowering response to vernalization in A. alpina.

Lower AaSPL15 mRNA levels in young compared with older plants suggested that miR156 influences vernalization through AaSPL15. To test this idea, we generated Paj plants that carried rotAaSPL15-VN or rAaSPL15-VN, which expresses a miR156-resistant form of AaSPL15 mRNA (fig. S6). Confocal microscopy revealed that 8-weekold retAaSPL15-VN accumulated VENUS:AaSPL15 protein during vernalization at the SAM, but that 3-week-old plants did not (Fig. 2A and fig. S7). By contrast, in rAaSPL15-VN plants, VENUS:AaSPL15 accumulated at the SAM of plants of both ages during vernalization (Fig. $2 \mathrm{~A}$ ). Thus, the sensitivity to miR156 ensures that SPL15 only accumulates at the SAM of adult plants. Moreover, young $r A a S P L 15-V N$ flowered in response to vernalization, whereas young wtAaSPL15-VN did not (Fig. 2B and fig. S8), indicating that SPL15 expression is sufficient to trigger flowering of young plants during vernalization. Thus, the antagonistic regulation of miR156 and SPL15 expression during cold determines the age at which A. alpina can respond to vernalization.

The resistance of SPL15 to miR156 also modified the flowering response of lateral branches (Fig. 2C). Axillary meristems of $A$. alpina attain competence to flower dependent on their age and independent of the $\operatorname{SAM}(8,20)$, so that after vernalization, lateral branches remain vegetative or flower in a predictable spatial pattern (Fig. 2D) $(3,21)$. Accumulation of VENUS:AaSPL15 varied among axillary meristems of wtAaSPL15-VN exposed to vernalization. The fusion protein accumulated only in meristems of lateral shoots old enough to have produced several leaves before vernalization (Fig. 2E), correlating with flowering response of these branches (fig. S9). By contrast, $r A a S P L 15-V N$ accumulated VENUS: 
AaSPL15 similarly in all axillary meristems, irrespective of the age of the lateral shoot (Fig. $2 \mathrm{E}$ and fig. S9). Also, a higher proportion of axillary meristems transitioned to flowering in rAaSPL15$V N$ (Fig. 2F). In rice, the closest homolog of SPL15 determines tiller bud outgrowth $(22,23)$, but comparison of bud dormancy in rAaSPL15-VN and wtAaSPL15-VN (Fig. 2F) plants suggested that in A. alpina, axillary bud outgrowth is controlled independently of AaSPL15. Thus, the miR156-SPL15 module contributes to polycarpic development of $A$. alpina by conferring competence to flower independently on individual meristems.

Whether SPL15 participates in the vernalization response of $A$. thaliana remains unclear because the annual plant responds to vernalization independently of age (20). We detected that SPL15 in A. thaliana is regulated in a manner similar to that described above in A. alpina. Thus, in 2-week-old FRI-Col, FLC down-regulation during vernalization was insufficient for VENUS: SPL15 accumulation because of high miR156 levels (Fig. 3, A and B, and figs. S10 and S11). Nevertheless, vernalization promoted flowering of these young plants. However, the transition took place only after returning to warm long days (Fig. 3C and fig. S12), which possibly involves the photoperiodic FLOWERING LOCUS T (FT) pathway. Accordingly, knockout alleles of FT and its paralogue TWIN SISTER OF FT (TSF) delayed flowering of 2-week-old FRI-Col exposed to vernalization (Fig. 3D). By contrast, 6-week-old FRI-Col, which contains low miR156 levels, ac- cumulated VENUS:SPL15 during vernalization (Fig. 3A and fig. S11). The adult plants transitioned to flowering in cold (Fig. 3C and fig. S12), and $F T$ no longer influenced flowering under these conditions (Fig. 3D and fig. S12). These data suggest that SPL15 and FT represent two independent pathways to flowering, and $A$. thaliana does not display age-related vernalization because on exposure to long days after vernalization, FT bypasses requirement for SPL15. Supporting this conclusion, spl15 ft tsf triple mutants in Col flowered late compared with either spl15 or $f t$ tsf mutants (Fig. 3E).

This model suggests that flowering of A. alpina in response to vernalization only occurs through the SPL15-dependent pathway. In A. thaliana, FLC directly represses FT transcription (24). Similarly, PEP1 in A. alpina down-regulated AaFT1 and AaFT3 (figs. S13 and S14), which can promote flowering (fig. S15). However, PEP1 is only repressed in cold, whereas $F L C$ remains stably repressed after vernalization $(3,4,25)$. Therefore, PEP1 may prevent flowering through $A a F T s$ after vernalization. Accordingly, ectopic $A a F T 1$ expression promoted flowering in A. alpina without cold exposure and independent of AaSPL15 expression (fig. S15). To test whether differences in PEP1/FLC regulation between annuals and perennials affected flowering responses to cold, we used material derived from an interspecies cross between A. alpina and its annual relative Arabis montbretiana (26). Similar to FLC in A. thaliana, its ortholog in A. montbretiana (AmFLC) is stably repressed after vernalization, and this species re- sponds to vernalization soon after germination (26). Back-crossing the interspecies hybrids to A. alpina, we derived two introgression lines, PEP1_IL and AmFLC_IL, that possess comparable genomic structures except for the region containing PEP1 or AmFLC (Fig. 4A), and neither flowered without vernalization (fig. S16). However, in the hybrid plants, $A m F L C$ remains stably repressed after vernalization, whereas PEP1 is reactivated (26). We found that 2-week-old AmFLC_IL flowered in response to vernalization, whereas 2-week-old PEP1_IL did not (Fig. 4B and fig. S16). Thus, the age-related repression of vernalization response in young plants was compromised in AmFLC_IL. That AmFLC conferred this difference was confirmed by introducing AmFLC locus into A. alpina pep1 mutant via Agrobacterium-mediated transformation, which recapitulated the phenotype of $A m F L C$ IL (Fig. 4, B and C, and fig. S17). Young AmFLC pepI showed neither AaSPL15 mRNA accumulation nor flower formation in cold (Fig. 4, D and E, and fig. S18) but, as observed in A. thaliana, flowered after vernalization in response to long days, probably through $A a F T$ activation (Fig. 4F and fig. S17). Thus, the stable repression of $A m F L C$ after vernalization reconstructs the annual flowering response to winter cold in perennial A. alpina.

We show here that the age-dependent response to vernalization in perennial $A$. alpina occurs through SPL15 (fig. S19). Reactivation of PEP1 in warm ensures that the FT pathway does not promote flowering after vernalization (fig. S20).
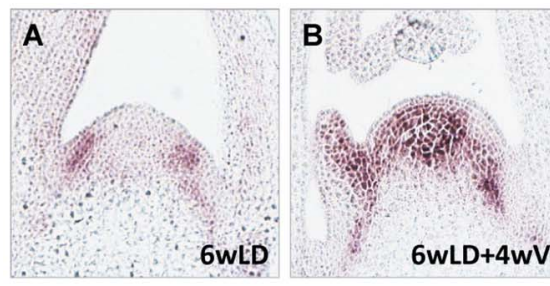

E

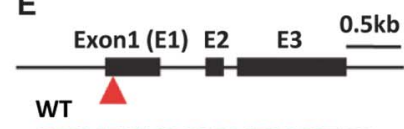

CTGAATCAGACAGAGTCAGGTGG Aaspl15-rg3 (-1bp)

CTGAATCAGACAGAGT - AGGTGG

Aaspl15-rg4 (-4bp)

CTGAATCAGACAG--- - AGG $\underline{\text { GG }}$

Aaspl15-rg5 (+1bp)

CTGAATCAGACAGAGTCCAGG $\underline{\underline{T G G}}$

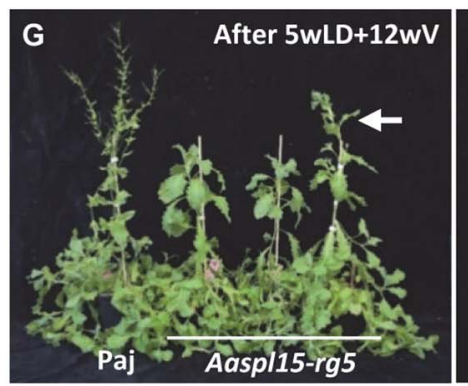

\begin{abstract}
F
\end{abstract}

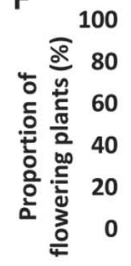

C

2wLD

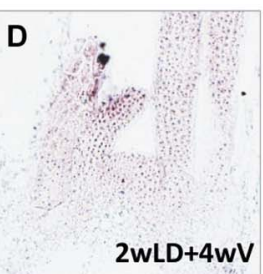

$2 w L D+4 w V$

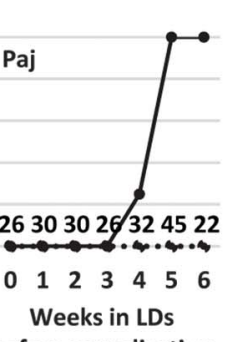

before vernalization

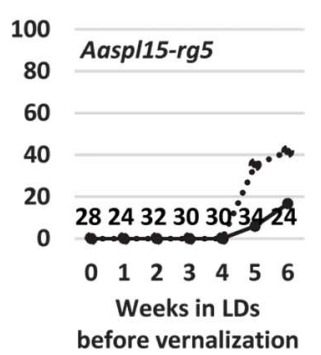

Fig. 1. AaSPL15 is a crucial component of the vernalization mechanism of $\boldsymbol{A}$. alpina. (A to D) AaSPL15 mRNA accumulation at shoot apices of $A$. alpina Paj before and after 4-week vernalization. Plants were grown under long days (LDs) for 6 or 2 weeks before vernalization. (E) Aaspl15-knockout alleles induced by use of CRISPR/Cas9. The arrowhead indicates the site targeted for mutagenesis. Twenty nucleotides homologous to the sgRNA and the PAM motif are marked with red letters and black underlined letters, respectively. (F) Vernalization response of Paj and Aaspl15-rg5 at different ages. Solid and dashed lines indicate the proportion of plants flowering or reverted from flower formation back to vegetative growth, respectively. The number of analyzed plants are indicated. (G to I) Defects in the vernalization response of Aasp/15 mutants. Arrows indicate $[(\mathrm{G})$ and $(\mathrm{I})]$ the plants that showed reversion and $(H)$ siliques formed on the reverted shoot. Plants at (G) 1 month and (I) 4 months after vernalization treatment are presented.
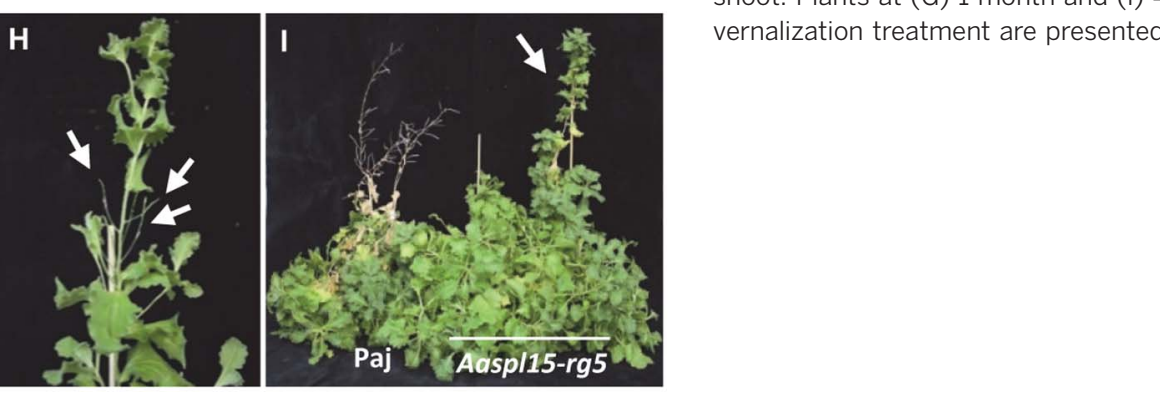
A

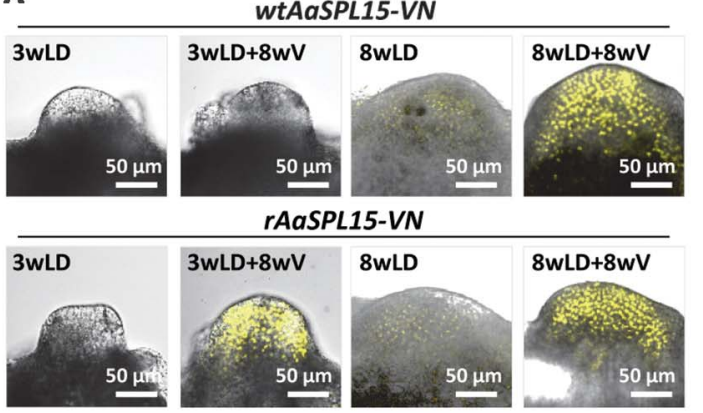

D

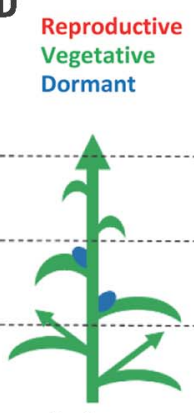

Before vernalization

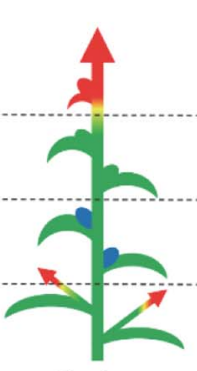

During vernalization

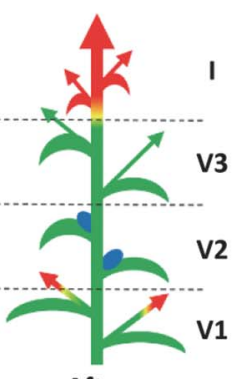

After

Fig. 2. AaSPL15 defines the competence of shoot meristems to flower in response to vernalization. (A) VENUS:AaSPL15 protein accumulation before and during vernalization at main shoot apices of plants of different ages carrying the wtAaSPL15-VN or rAaSPL15-VN transgenes. (B and $\mathbf{C}$ ) Earlier acquisition of sensitivity to vernalization in rAaSPL15-VN. Arrows indicate vegetative axillary branches formed after vernalization.

(D) Schematic showing developmental fates of axillary meristems at different

B

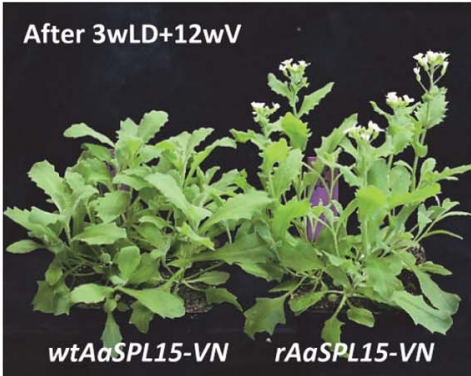

$\mathbf{E}$

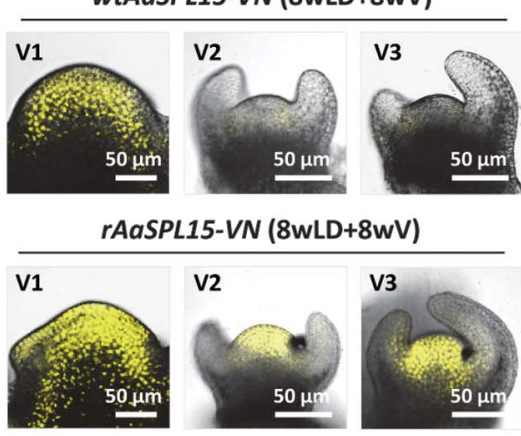

C

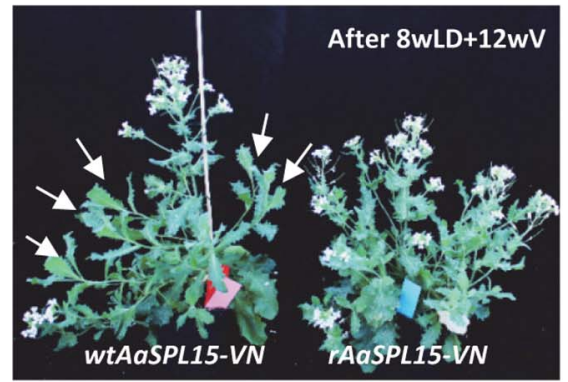

$\mathbf{F}$

Feproductive Vegetative Dormant
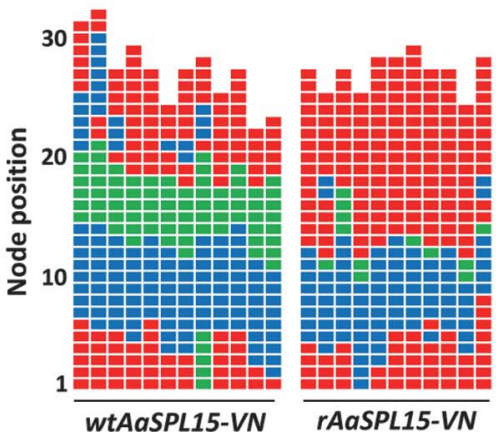

A
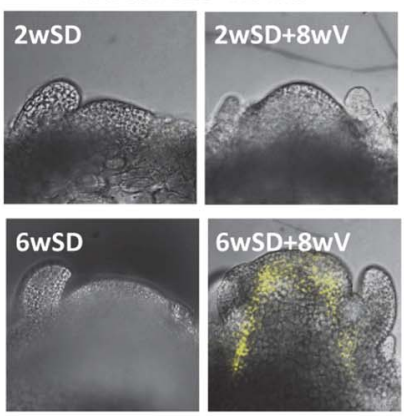

rAtSPL15-VN FRI
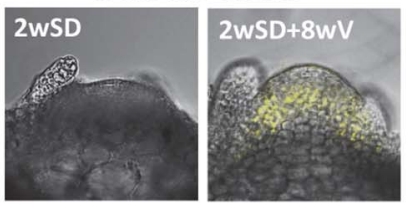

B
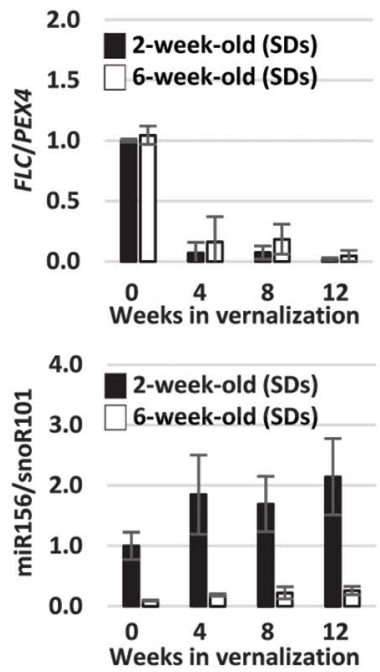

C

positions on the shoot of A. alpina. Arrows and ovals represent shoots and buds, respectively. (E) Accumulation of VENUS:AaSPL15 at axillary meristems of wtAaSPL15-VN and rAaSPL15-VN during vernalization. V1 to V3 indicate the positions on the shoot illustrated in (D). (F) Quantification of developmental fates of axillary meristems of wtAaSPL15-VN and rAaSPL15-VN at 6 weeks after exposure of 8-week-old plants to 12-week vernalization $(8 w L D+12 w V)$. Each column depicts an individual plant.
Fig. 3. FT bypasses the requirement for SPL15 in cold-induced flowering of young $\boldsymbol{A}$. thaliana. (A) Accumulation of VENUS:SPL15 in wtAtSPL15-VN FRI and rAtSPL15-VN FRI before and during vernalization. (B) FLC mRNA and miR156 levels at the shoot apex of FRI-Col. The expression levels at 2 weeks old are set to 1.0 for normalization. The error bars indicate standard deviation of three biological replicates. (C) $L F Y$ mRNA accumulation in FRI-Col exposed to vernalization at 2WSD and 6wSD. (D) A lack of FT activity 2-week-old (SDs)

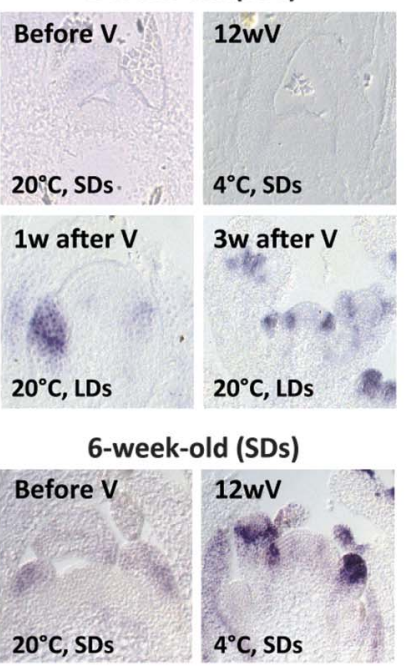

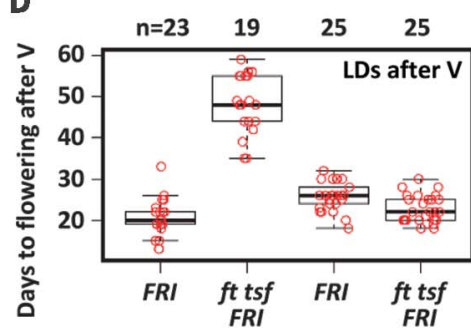

$\overline{2 w S D+12 w V} \overline{6 w S D+12 w V}$

E

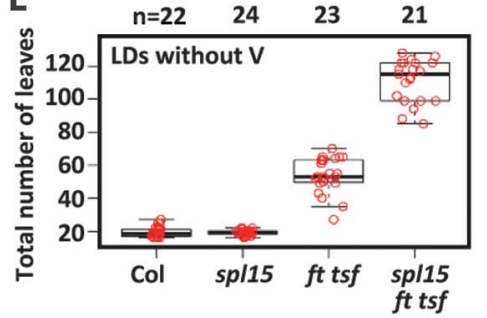

delays flowering of FRI-Col exposed to vernalization at 2wSD but not when exposed at 6wSD. (E) SPL15 and FT represent independent pathways to flowering on the basis of a synergistic genetic interaction under LDs. Flowering time is presented as (D) days to flowering after vernalization $(\mathrm{V})$ and $(\mathrm{E})$ total leaf number. In the box plots, the interquartile range box represents the middle $50 \%$ of the data. Whiskers extend 1.5 times the interquartile range and the center line shows the median. 

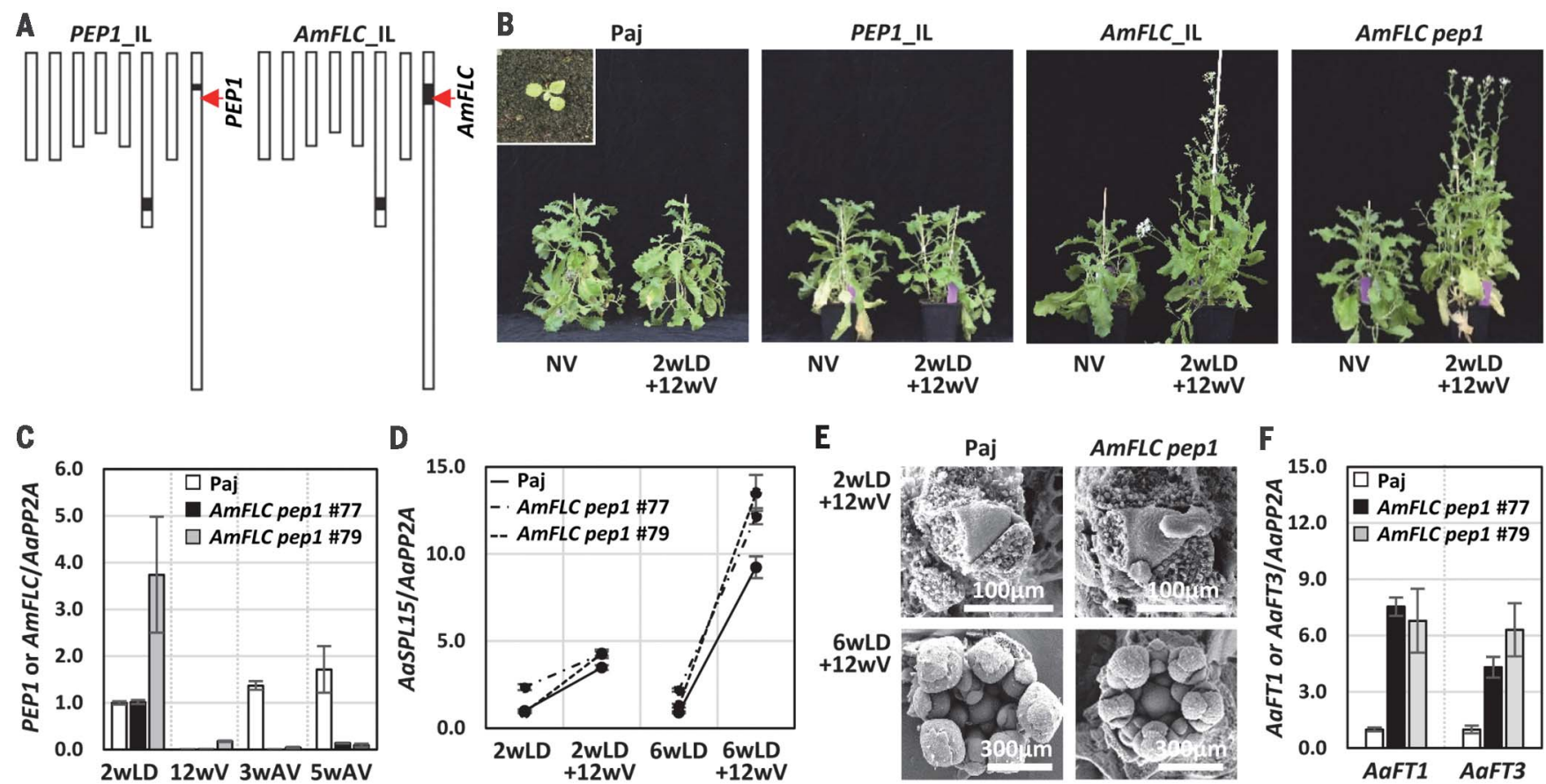

Fig. 4. Reconstruction of the annual response to age in perennial A. alpina by introducing AmFLC. (A) Schematic genome structure of PEP1_IL and AmFLC_IL. Black boxes indicate the introgressed A. montbretiana genomic segments. (B) Flowering response of Paj, PEP1_IL, AmFLC_IL, and AmFLC pep1 exposed to cold as a 2-week-old seedling (inset). (C and D) FLC orthologs (C) and AaSPL15 (D) mRNA levels at shoot apices of Paj and AmFLC pep1. AV, after vernalization. (E) Scanning electron microscopy of shoot apices

of Paj and AmFLC pep1 immediately after vernalization treatment. Neither Paj nor AmFLC pep1 produced flowers during cold if exposed to vernalization as a young seedling $(2 w L D+12 w V)$, but both did so if exposed to vernalization as an older plant $(6 w L D+12 w V)$. (F) AaFT1 and AaFT3 mRNA levels in leaves of Paj and AmFLC pep1 at 5WAV. The expression levels in [(C) and (D)] Paj at 2wLD and (F) Paj are set to 1.0 for normalization. Error bars indicate standard deviation of two biological replicates.

Despite the SPL15 pathway and its regulation being conserved, annual $A$. thaliana is not dependent on SPL15 because the stable repression of $F L C$ allows young plants to flower through FT after vernalization. Redundancy between SPL15 and FT may explain variation in severity of the spl15 late-flowering phenotype (16, 17). Also, several Scandinavian accessions of $A$. thaliana require vernalization to flower and additionally carry hypomorphic FT alleles (27), suggesting that when exposed to short growing seasons, even annuals may benefit from reduced photoperiodic pathway activity and consequent increased dependency on SPL15. Thus, the ability to shift the balance between these pathways confers versatility in flowering physiology both within a species and during species divergence. These buffered signaling pathways enable flexible responses to shifting environments and diversification in life-history evolution $(28,29)$.

\section{REFERENCES AND NOTES}

1. F. Andrés, G. Coupland, Nat. Rev. Genet. 13, 627-639 (2012).

2. Y. Hyun, R. Richter, G. Coupland, Plant Physiol. 173, 36-46 (2017),

3. R. Wang et al., Nature 459, 423-427 (2009).

4. S. D. Michaels, R. M. Amasino, Plant Cell 11, 949-956 (1999).
5. C. C. Sheldon et al., Plant Cell 11, 445-458 (1999).

6. C. M. Zhou et al., Science 340, 1097-1100 (2013).

7. P. Baduel, B. Arnold, C. M. Weisman, B. Hunter, K. Bomblies, Plant Physiol. 171, 437-451 (2016)

8. S. Bergonzi et al., Science 340, 1094-1097 (2013).

9. J. W. Wang, B. Czech, D. Weigel, Cell 138, 738-749 (2009).

10. G. Chuck, A. M. Cigan, K. Saeteurn, S. Hake, Nat. Genet. 39, 544-549 (2007).

11. G. Wu et al., Cell 138, 750-759 (2009).

12. R. Wang et al., Plant Cell 23, 1307-1321 (2011).

13. W. Deng et al., Proc. Natl. Acad. Sci. U.S.A. 108, 6680-6685 (2011)

14. J. L. Mateos et al., Genome Biol. 16, 31 (2015).

15. J. L. Mateos et al., Proc. Natl. Acad. Sci. U.S.A. 114 E11037-E11046 (2017).

16. M. Xu et al., PLOS Genet. 12, e1006263 (2016).

17. Y. Hyun et al., Dev. Cell 37, 254-266 (2016).

18. M. Cartolano et al., Proc. Natl. Acad. Sci. U.S.A. 112, 10539-10544 (2015)

19. Y. Hyun et al., Planta 241, 271-284 (2015).

20. J. Y. Park, H. Kim, I. Lee, Sci. Rep. 7, 13348 (2017).

21. A. Lazaro, E. Obeng-Hinneh, M. C. Albani, Plant Physiol. 176, 2819-2833 (2018)

22. Y. Jiao et al., Nat. Genet. 42, 541-544 (2010).

23. K. Miura et al., Nat. Genet. 42, 545-549 (2010)

24. I. Searle et al., Genes Dev. 20, 898-912 (2006).

25. A. R. Gendall, Y. Y. Levy, A. Wilson, C. Dean, Cell 107, 525-535 (2001).

26. C. Kiefer et al., Mol. Ecol. 26, 3437-3457 (2017)

27. A. Strange et al., PLOS ONE 6, e19949 (2011).

28. J. L. Hartman 4th, B. Garvik, L. Hartwell, Science 291 1001-1004 (2001)
29. S. van Wageningen et al., Cell 143, 991-1004 (2010)

\section{ACKNOWLEDGMENTS}

We thank M. Albani, F. Turck, M. Romera, D. Ó'Maoiléidigh, A. Pajoro, and A. van Driel for their critical reading of the manuscript. Funding: This work was funded through European Research Council grant HyLife as well as by Deutsche Forschungsgemeinschaft through SPP1530 and Excellence Cluster CEPLAS. The laboratory of G.C. receives core funding from the Max Planck Society. Author contributions: Y.H. and G.C. initiated and coordinated the project and wrote the manuscript. Y.H.

C.V., V.T., S.B., R.R., and R.M.-G. performed the experiments. C.K. and E.S. contributed to the interspecies hybrid construction and bioinformatic data analysis, respectively. Competing interests: None declared. Data and material availability: All data needed to evaluate the conclusions in the paper are present in the paper or the supplementary materials. The Aaspl15 mutants, ft tsf spl15 mutants,

SPL15 A. alpina transgenic plants, and all plasmid constructions using AaSPL15 are available from G.C. under a materials transfer agreement with the Max Planck Institute for Plant Breeding Research.

\section{SUPPLEMENTARY MATERIALS}

www.sciencemag.org/content/363/6425/409/suppl/DC1

Materials and Methods

Figs. S1 to S20

Tables S1 and S2

References (30-38)

24 July 2018; accepted 19 December 2018

10.1126/science.aau8197 


\section{Science}

\section{A regulatory circuit conferring varied flowering response to cold in annual and perennial plants}

Youbong Hyun, Coral Vincent, Vicky Tilmes, Sara Bergonzi, Christiane Kiefer, René Richter, Rafael Martinez-Gallegos, Edouard Severing and George Coupland

Science 363 (6425), 409-412.

DOI: $10.1126 /$ science.aau8197

\section{Diversity in flowering regulation}

Annual plants flower for one season and then die, whereas perennials can flower repeatedly year after year. Hyun et al. explain how different signaling pathways control such variation in flowering. The perennial pathway requires a floral integrator limited to older shoots. The annual pathway, on the other hand, allows a photoperiodic response to incite flowering on young shoots. Solutions to challenging environments may emerge through evolution as the balance between these regulatory systems shifts.

Science, this issue p. 409

ARTICLE TOOLS

SUPPLEMENTARY

MATERIALS

REFERENCES

PERMISSIONS http://science.sciencemag.org/content/363/6425/409

http://science.sciencemag.org/content/suppl/2019/01/23/363.6425.409.DC1

This article cites 38 articles, 15 of which you can access for free http://science.sciencemag.org/content/363/6425/409\#BIBL

http://www.sciencemag.org/help/reprints-and-permissions

Use of this article is subject to the Terms of Service

Science (print ISSN 0036-8075; online ISSN 1095-9203) is published by the American Association for the Advancement of Science, 1200 New York Avenue NW, Washington, DC 20005. The title Science is a registered trademark of AAAS.

Copyright (C) 2019 The Authors, some rights reserved; exclusive licensee American Association for the Advancement of Science. No claim to original U.S. Government Works 